\title{
Enhanced Piezoelectric Coefficient of PVDF-TrFE Films via In Situ Polarization
}

\author{
Xiaoran $\mathrm{Hu}^{1,2}$, Mengli You ${ }^{1,2}, \mathrm{Na} \mathrm{Yi}{ }^{1,2}$, Xiaokun Zhang ${ }^{1,2 *}$ and Yong Xiang ${ }^{1,2,3}$ \\ ${ }^{1}$ School of Materials and Energy, University of Electronic Science and Technology of China, Chengdu, China, ${ }^{2}$ Advanced Energy \\ Institute, University of Electronic Science and Technology of China, Chengdu, China, ${ }^{3}$ Sichuan Flexible Display Materials Genome \\ Engineering Center, Chengdu, China
}

The $\mathrm{d}_{33}$ coefficient $=28 \mathrm{pC} / \mathrm{N}$ of PVDF-TrFE piezoelectric films was achieved by the in situ polarization. Compared with traditional poling methods, the in situ polarization is performed with low poling voltage and short poling time, and it can ensure the PVDFTrFE film with enhanced piezoelectric performances and uniform distribution among a large area of $200 \mathrm{~mm}^{2} \times 200 \mathrm{~mm}^{2}$. The processing influence of drying, annealing, and poling on the crystalline properties and piezoelectric performances were investigated. Besides, the obtained PVDF-TrFE films present a good piezoelectric response to different extents of mechanical stimulations, which have great potential in energy harvesting applications.

\section{OPEN ACCESS}

Edited by:

Sheng S. Zhang,

United States Army Research

Laboratory, United States

Reviewed by:

Mohd Faizul Mohd Sabri,

University of Malaya, Malaysia

Ryszard Wycisk,

Vanderbilt University, United States

*Correspondence:

Xiaokun Zhang

diankeda20201026@163.com

Specialty section:

This article was submitted to Electrochemical Energy Conversion and Storage,

a section of the journal Frontiers in Energy Research

Received: 26 October 2020

Accepted: 30 June 2021

Published: 10 August 2021

Citation:

HuX, You M, Yi N, Zhang X and Xiang Y (2021) Enhanced Piezoelectric Coefficient of PVDF-TrFE Films via In

Situ Polarization.

Front. Energy Res. 9:621540. doi: 10.3389/fenrg.2021.621540
Keywords: PVDF-TrFE, in situ polarization, D 33, piezoelectricity, nanogenerators

\section{INTRODUCTION}

Since Kawai initially reported the poly(vinylidene fluoride) (PVDF) in 1969 (Moyle, 1969), PVDF has attracted considerable attention (Furukawa, 1989; Bune et al., 1998) owing to its unique piezoelectricity. The piezoelectricity allows it to convert the ambient mechanical stimulations to electricity, which has been widely used in energy harvesting applications such as the piezoelectric nanogenerators (PENGs). Recently, the PENGs are used as power sources to drive the low-power wearable devices. With the rapid development of smart wearable devices, the energy conversion efficiency, flexibility, and transparency are the most important features of PENGs. As the most studied polymer for PENG, PVDF-TrFE has relative low remnant polarization and piezoelectric coefficient but irreplaceable flexibility and transparency (Chan et al., 2003; Pi et al., 2014; Mcginn et al., 2020). Thus, it is vital to improve the piezoelectric properties of PVDF to promote its value in the smart wearable device applications.

The piezoelectricity of PVDF originates from its crystalline structures. As a semicrystalline polymer, PVDF presents a complex crystalline structure and crystalline phases (commonly $\alpha-, \beta-$, and $\gamma_{\text {-) }}$ (Dai et al., 2016). The nonpolar $\alpha$-phase has a TGTG chain conformation, resulting in the self-cancelation of dipoles (Lovinger et al., 1983). The $\beta$-phase has an all trans (TTTT) planar zigzag with the strongest polarity, while the $\gamma$-phase (Hasegawa et al., 1972; Masahiko et al., 1977; Lovinger, 1983; Sajkiewicz et al., 1999) displays a $\mathrm{T}_{3} \mathrm{GT}_{3} \mathrm{G}$ chain conformation which is partially polar. However, the $\alpha$-phase is the most favorite form in thermodynamics with nonpolar crystalline phases. By thermal treatment (Prest and Luca., 1978) or poling under a high electric field $(150 \mathrm{kV} / \mathrm{mm})$ (Davis et al., 1978; Banik et al., 1980), the $\alpha$-phase can be transformed to the $\gamma$-phase or $\beta$-phase.

In order to increase the $\beta$-phase and simultaneously reduce the crystallinity of the $\alpha$-phase, many methods have been developed during the preparation of PVDF materials. Copolymers of PVDF, such as $\mathrm{P}(\mathrm{VDF}-\mathrm{TrFE})$, have been synthesized to achieve an intrinsic $\beta$-phase crystal. Besides, special 
treatment including electrospinning ( $\mathrm{Li}$ et al., 2008), solventcasting (Hu et al., 2015), mechanical stretching, and polarization under high electric fields (Fukada and Sakurai., 1971; Cheng-Lu et al., 2014) of PVDF (Sencadas et al., 2009; Sharma et al., 2013; Won et al., 2016) have been employed. According to the research report on the electrospinning process of the high $\beta$-phase PVDF membrane, the micro-patterned device as an energy harvester can generate an open circuit voltage density of $1.42 \mathrm{~V} \mathrm{~m}^{-2}$ (Fadzallah et al., 2020). However, the electrospinning and solvent-casting usually induce some undesired structure deformation or microstructure defeats. And additional requirement such as long-lasting heating or poling may impede the large-area processing and uniform distribution of PVDF films. As the most used method, electrical poling faces a problem such that when the thickness of the film is low ( $\mu \mathrm{m}$ or thinner), the leakage current may cause breakdown during the poling. Thus, alternative methods are needed to improve the processing efficiency and uniformity.

In this study, a large area of $200 \mathrm{~mm} \times 200 \mathrm{~mm}$ of the PVDFTrFE film with enhanced piezoelectric coefficients was prepared via in situ polarization. The in situ polarization has shorter processing time than the traditional methods. The prepared PVDF-TrFE films have good piezoelectric performance with great distribution uniformity. The crystalline structure and the piezoelectric properties of the $\mathrm{P}(\mathrm{VDF}-\mathrm{TrFE})$ on different processes were investigated. It was found that the in situ poling process can enhance the crystalline structure of the $\beta$-phase and generate the preferred orientation of its polymer chain.

\section{EXPERIMENT}

\section{Materials}

The materials were used with no further purification and purchased from the following companies: $\mathrm{P}(\mathrm{VDF}-\mathrm{TrFE})$ (FC20, 80/20 mol\%, Piezotech SAS, France) and methyl ethyl ketone (MEK, 99.0\%, KESHI, Chengdu, China).

\section{METHODS}

Typically, $5.52 \mathrm{~g}$ of PVDF-TrFE was dissolved in $40 \mathrm{ml}$ of MEK and stirred by a magnetic stirrer for $5 \mathrm{~h}$. Then, $3 \mathrm{ml}$ of the solution was slit-coated on the ITO glasses and was transferred to a vacuum plate to evaporate the solvent and form the PVDFTrFE films with thickness of $10 \mu \mathrm{m}$. These films were further annealed at $140^{\circ} \mathrm{C}$ for $1 \mathrm{~h}$. Subsequently, the PVDF-TrFE films were in situ poled by a laboratory-made equipment. The preparation process was shown in Figure 1. For electrical measurements, Ag was vacuum-sputtered on both sides of the films to form electrodes and compose a metal-insulator-metal structure. Plastic is used as the sealing cover of the PVDF-TrFE device.

The morphology of the PVDF-TrFE films was observed by a field-emission scanning electron microscope (SEM, S-3400, Hitachi). X-ray diffraction (XRD) was carried out on a
D/Max2500 VB2t/PC X-ray diffractometer (Rigaku, Japan) with a $\mathrm{Cu}$ target radiation for a $2 \theta$ range of $5-50^{\circ}$ at an angular resolution of $0.5^{\circ}$. The differential scanning calorimetry (DSC) test was performed with Mettler-Toledo DSC instrument under nitrogen to heat the film to $200^{\circ} \mathrm{C}$ at $10^{\circ} \mathrm{C} / \mathrm{min}$. The piezoelectric coefficients of the PVDF-TrFE film sample tested by piezoelectric tester $\left(d_{33}\right.$ METER, YE2730A, APC) was introduced. The energy harvesting performances of PVDF-TrFE devices were tested under the repeated impact with different accelerated speeds drove by the NTI AG HS01-37 × 166 linear motor, and the output voltage or current generated simultaneously were collected using a Keithley 6,514 system electrometer and a Stanford Research SR570 low-noise current preamplifier. The energy harvesting experiment diagram is shown in Figure 2.

\section{RESULTS AND DISCUSSION}

Figure 3 shows the schematic diagram of the in situ polarization process used in the study. The laboratory-made polarization equipment primarily consisted of source, grid, and rotated stage. After the source and grid move above the sample, a voltage of $7.5 \mathrm{kV}$ was applied on the source electrode to generate electronegativity ions; then, these electronegativity ions were accelerated by the grid with a voltage of $3.0 \mathrm{kV}$ and deposited on the surface of the sample evenly. The rather close distance (equal to the thickness of film, $15 \mu \mathrm{m}$ ) between the virtual electrode formed by the electronegativity ions and the bottom electrode can generate a tremendous electrical field which leads to an effective polarization. During the poling process, the substrate rotated for $360^{\circ}$ slowly, which ensured the uniform piezoelectricity of the PVDF-TrFE coatings. The poling process only lasted for about $5 \mathrm{~min}$, which is much shorter than that reported in many other poling methods, as provided in Table 1.

After the in situ polarization, the piezoelectric coefficient $\left(d_{33}\right)$ of the PVDF-TrFE film is tested, and the result is shown in Table 2. The PVDF-TrFE films show rather low piezoelectricity $\left(d_{33}=0 \mathrm{pC} / \mathrm{N}\right)$ after drying in the oven. The value of $\mathrm{d}_{33}$ improved slightly to $2.5 \mathrm{pC} / \mathrm{N}$ after the PVDF-TrFE films annealed in $140^{\circ} \mathrm{C}$ for $1 \mathrm{~h}$ due to the formation of $\beta$-phase crystals caused by heat treatment. After poling, the PVDF-TrFE film showed dramatically enhanced piezoelectric coefficient of $\mathrm{d}_{33}=25 \mathrm{pC} / \mathrm{N}$. Then the $d_{33}$ constants slightly increased from 25 to $28 \mathrm{pC} / \mathrm{N}$ after 2 days. Our PVDF-TrFE possesses higher $\mathrm{d}_{33}$ coefficient than the $d_{33}$ coefficient of reported PVDF-based piezoelectric materials PVDF $\left(\mathrm{d}_{33}=20 \mathrm{pC} / \mathrm{N}\right)$ (Liu et al., 2017), PVDFPZT $\left(d_{33}=20 \mathrm{pC} / \mathrm{N}\right)$ (Chen et al., 2019). Besides, it has been reported that the residual electronegativity ions during the poling process may create an electric field which still improves the orientation of the dipole (Koten et al., 1987). And the piezoelectric properties of PVDF-TrFE coatings stayed stable in 6 months as we investigated. Besides, the piezoelectric coefficient of the $200 \mathrm{~mm}^{2} \times 200 \mathrm{~mm}^{2}$ PVDF-TrFE film after polarization was characterized by a $d_{33}$ mapping 


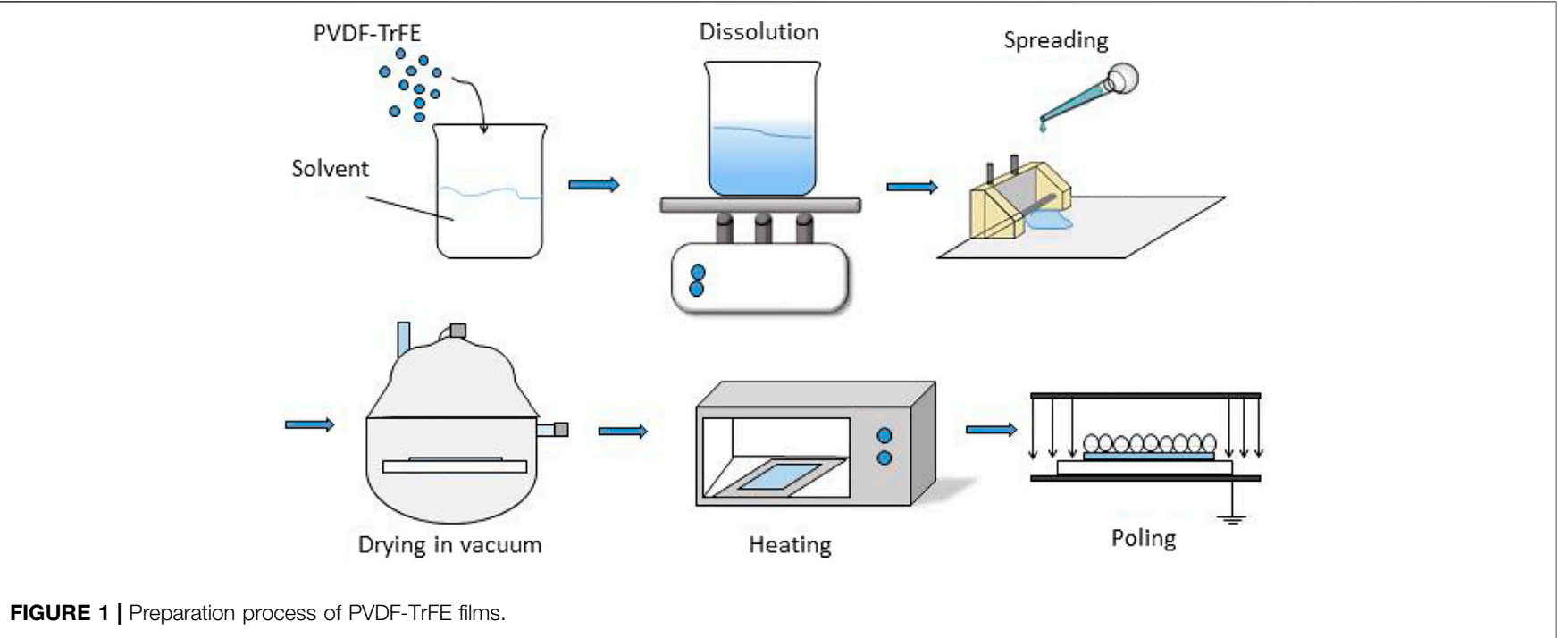

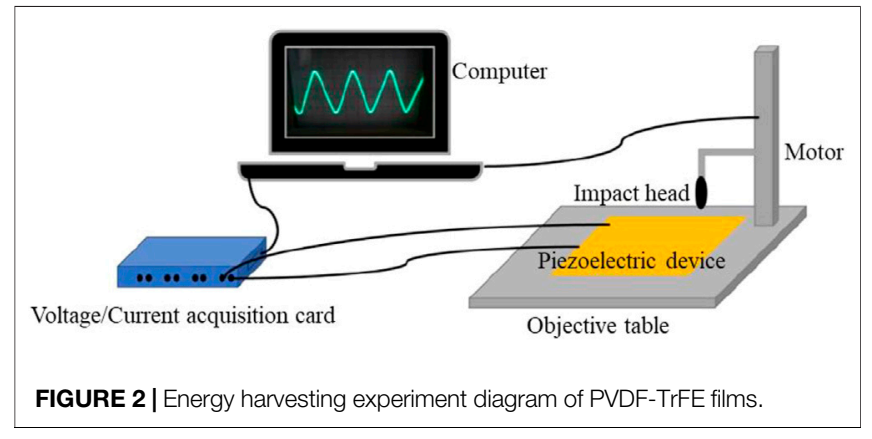

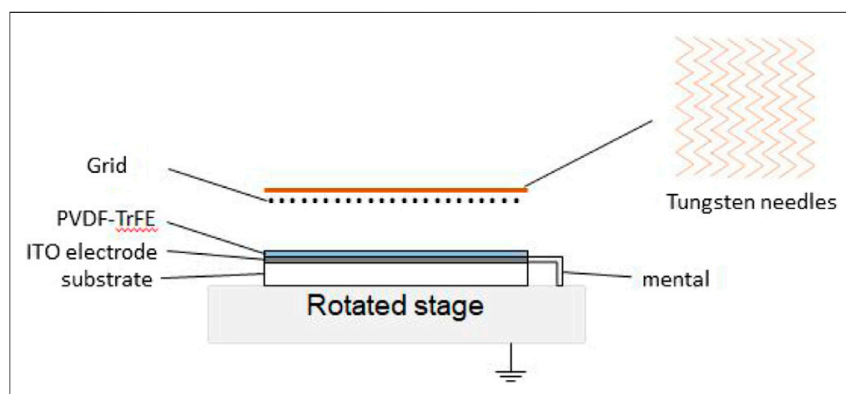

FIGURE 3 | Schematic diagram of in situ polarization.

detector, and the results are shown in Figure 4. The piezoelectric coefficient of the polarized film was $25 \pm$ $2 \mathrm{pC} / \mathrm{N}$, with excellent distribution.

The crystalline properties of PVDF-TrFE after drying, annealing, and poling were characterized by XRD, as provided in Figure 5. In Figure 5, the XRD patterns show that after drying the PVDF-TrFE films, two peaks at $18.27^{\circ}$ and $20.12^{\circ}$ were observed. The peak at $2 \theta=18.27^{\circ}$ is corresponding to the
TABLE 1 | Comparison of in situ polarization and common methods.

Methods

$\underset{\text { min }}{\text { Time/ }} \quad \operatorname{Temp}^{\circ} \mathrm{c}$

$\mathrm{MV} / \mathrm{m}$

In situ polarization

Electric-field (Bae and Chang, 2015;

$5 \quad-\quad 4,500-9,000$

Bhavanasi et al., 2015; Dietze and

Es-Souni, 2008; Pi et al., 2014; Yaqoob

and Chung, 2017; Ying et al., 2015)

Mechanical drawing (Alamusi et al., 2012) 5-30 60- 20-60

High pressure (Karan et al., 2016; Yaqoob

and Chung, 2017)
$5-$
TABLE 2 | Comparison of $d_{33}$ of PVDF-TrFE films after different processing stages.

\begin{tabular}{|c|c|}
\hline Item & d33/pC \\
\hline Drying & 0 \\
\hline Annealing & 2 \\
\hline Poling & 25 \\
\hline
\end{tabular}

(100) crystal planes of the $\alpha$-phase. Meanwhile, the $\gamma$-phase is present at the superposition point of the peaks located at $18.5^{\circ}$ associated with (020) crystal planes (Xue et al., 2013). Albeit $\alpha$ and $\gamma$-phase showed the same diffraction peaks around 18.3 , it was more likely attributed to the $\gamma$-phase (Nan et al., 2017). The sharp peak at $20.12^{\circ}$ represents the Bragg diffraction of (110)/ (200) of the $\beta$-phase (Ohigashi et al., 1995) and is from the ferroelectric $\beta$-phase having all-trans conformation. After annealing at $140^{\circ} \mathrm{C}$ for $1 \mathrm{~h}$, the only sharp peak at $20.12^{\circ}$, which referred to the ferroelectric $\beta$-phase, became more obvious. The calculated crystallinity of the sample after annealing is $75 \%$. There were two extremely weak and broad peaks at $2 \theta=35.51^{\circ}$ and $40.91^{\circ}$, respectively, which indicates a 


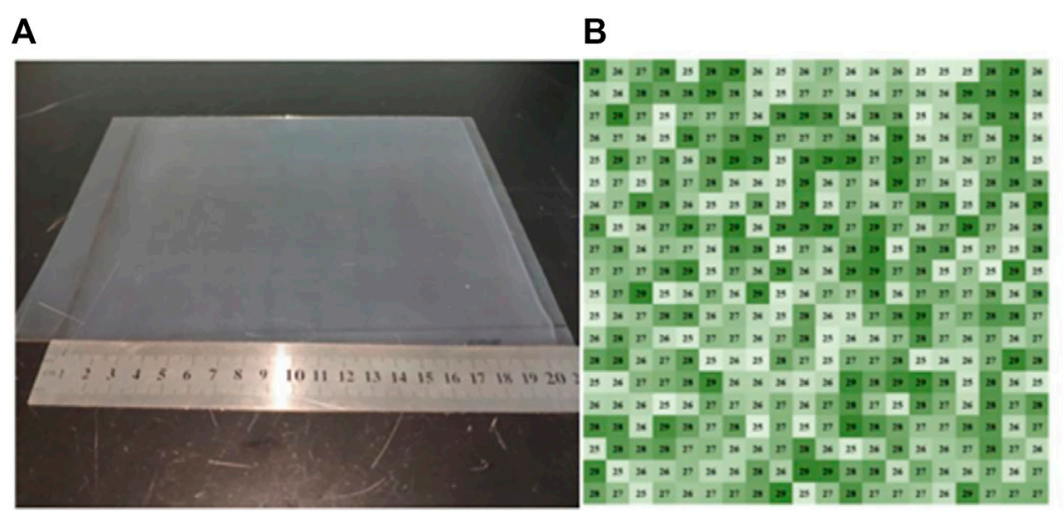

FIGURE 4 | (A) Large-area PVDF-TrFE polymer film and (B) mapping image of the polarized PVDF-TrFE film.

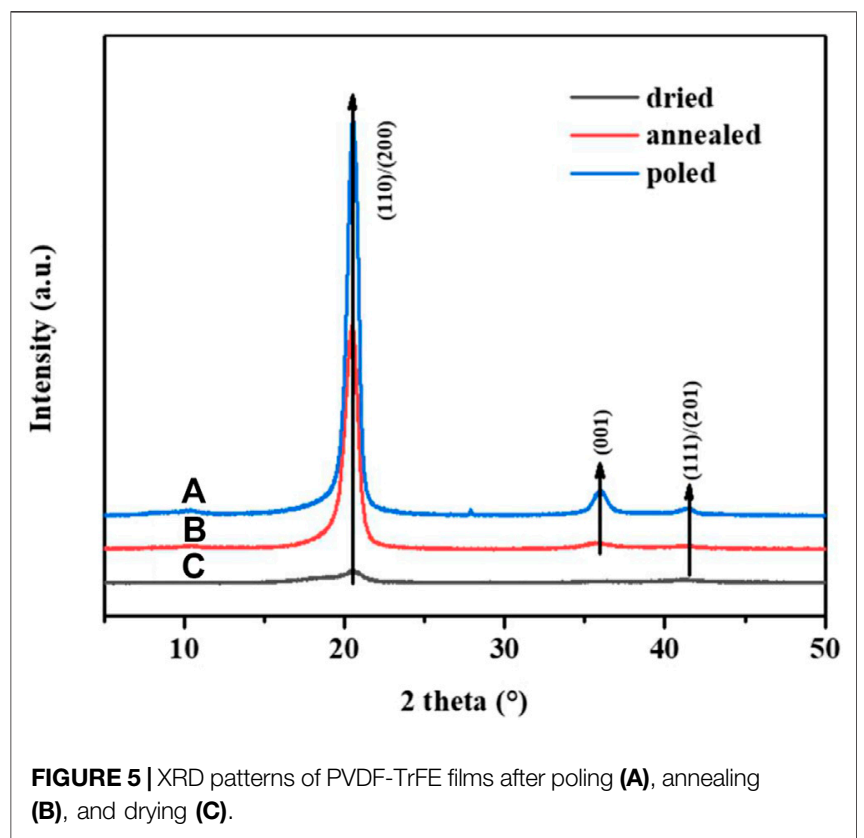

highly preferred orientation for the PVDF-TrFE films (Lutkenhaus et al., 2010). And the peak at $2 \theta=18.27^{\circ}$ vanished, indicating that the $\gamma$-phase disappeared in the high temperature. The calculated crystallinity of PVDF-TrFE films after the poling process is $77 \%$, which indicated the distinct $\beta$-phase peak was also found after polarization.

To further understand the crystalline behavior, DSC has been employed to characterize the crystalline behavior of different processing stages of PVDF-TrFE films. As shown in Figure 6, two peaks including a broad one at $120^{\circ} \mathrm{C}$ and a sharp one at $146^{\circ} \mathrm{C}$ were observed in the sample after drying. These two peaks are attributed to the $\mathrm{F}-\mathrm{P}$ transition $\left(\mathrm{T}_{\mathrm{C}}\right)$ and melting point $\left(\mathrm{T}_{\mathrm{m}}\right)$ of $\beta$-PVDF-TrFE, respectively. The imperfect ferroelectric phase and grains dispersing in large size range can be indicated from the broad temperature range of the $\mathrm{T}_{\mathrm{C}}$. After annealing at $140^{\circ} \mathrm{C}$ for $1 \mathrm{~h}$, the $\mathrm{T}_{\mathrm{C}}$ peak became sharper and gained an increased $\triangle \mathrm{H}_{\mathrm{C}}$

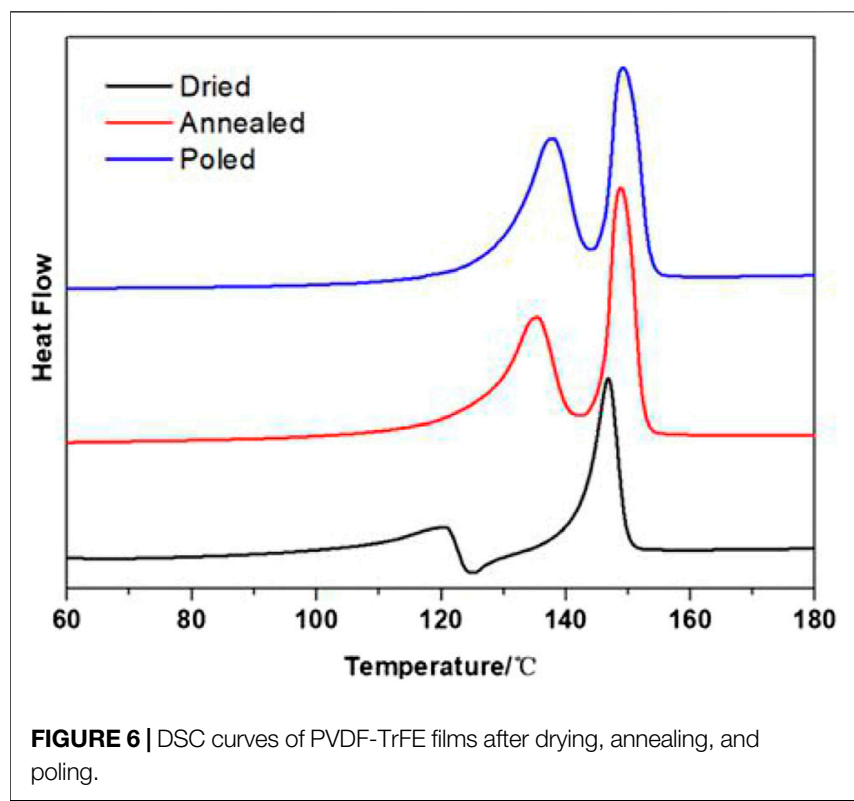

at about $14^{\circ} \mathrm{C}$ higher than that of the sample after drying. That indicates the annealing process improves the formation of large ferroelectric domains. As for the $\mathrm{T}_{\mathrm{m}}$ peak, melting and recrystallization of the crystal grains at $140^{\circ} \mathrm{C}$ led to higher and sharper peak with melting point increasing for $3^{\circ} \mathrm{C}$, which implied both crystallinity and crystal size are increased. The entire phenomenon can be attributed to the increased cohesion energy associated with a high melting point, which prevents the easy deformation of polymers.

The SEM of PVDF-TrFE films after drying, annealing, and poling is provided in Figure 7. It can be seen that the surface of PVDF-TrFE films dried in the vacuum oven was smooth in Figure 7A. After annealing at $140^{\circ} \mathrm{C}$ for $1 \mathrm{~h}$, the PVDF-TrFE films (Figure 7B) showed a lot of grains which were composed of randomly distributed $200-300 \mathrm{~nm}$ rod-like crystallites. This is a typical characteristic of the $\beta$-phase PVDF-TrFE. It has been reported that the content of TrFE can influence the crystalline 


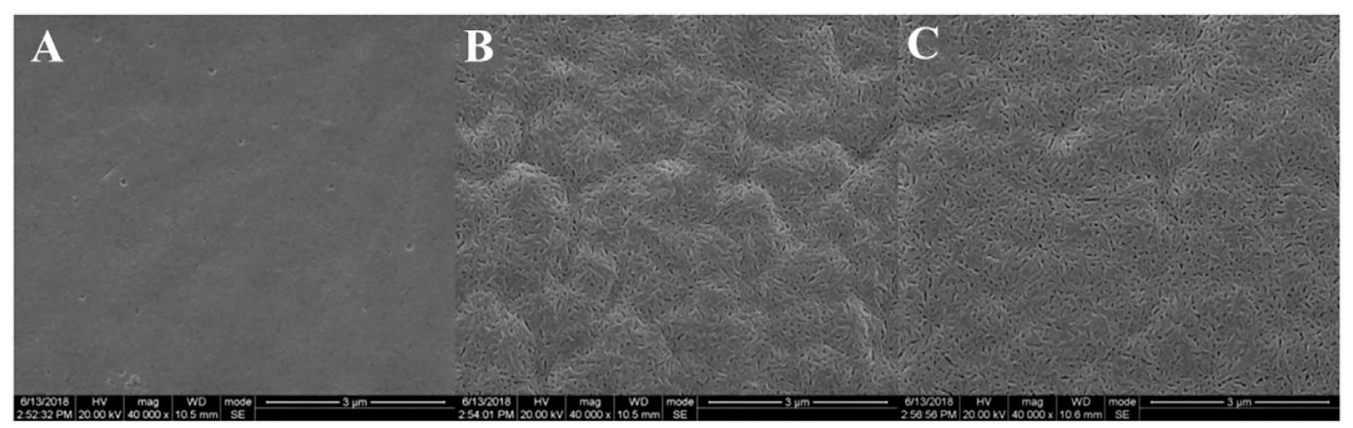

FIGURE 7 | SEM images of PVDF-TrFE after (A) drying, (B) annealing, and (C) poling.
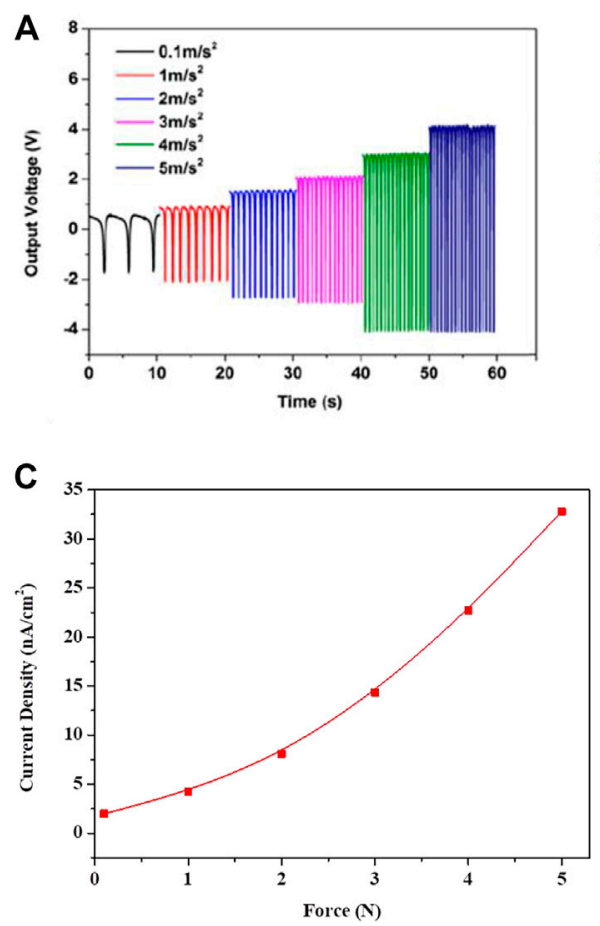

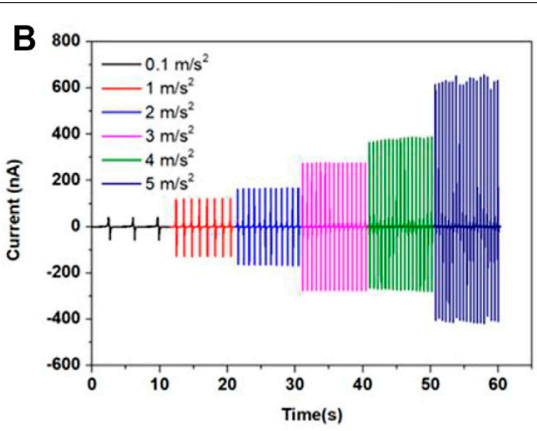

D

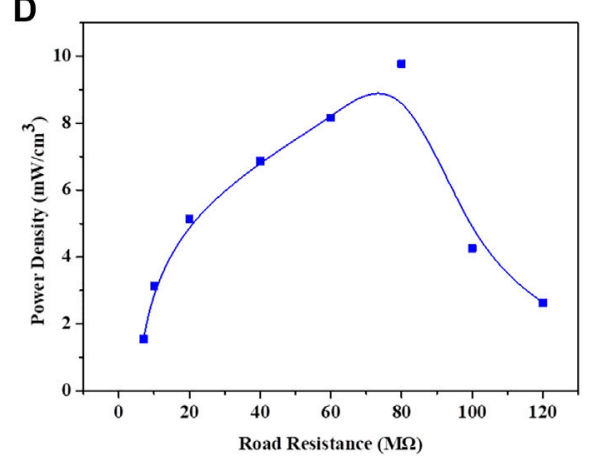

FIGURE 8 | (A,B) Output voltage and current of the PVDF-TrFE film under linear motor impact with different accelerated speeds. (C) Graph of the relationship between current density and applied stress. (D) The curves between power density and load resistance.

polymorphs of PVDF-TrFE. When the PVDF-TrFE content in TrFE is over $20 \mathrm{~mol} \%$, it can directly form the $\beta$-phase crystal (Wang et al., 2007). The image of PVDF-TrFE after poling (Figure 7C) showed more distinct grain than that of the PVDF-TrFE films after annealing. This proves the in situ polarization process leads a dramatic improvement of the formation of the $\beta$-phase crystal in PVDF-TrFE films.

The output performances of the PVDF-TrFE film were measured under linear motor impact with different accelerated speeds. Figure 8A and Figure 8B show the open-circuit voltage and short-circuit current of the mental/PVDF-TrFE/mental stacked piezoelectric devices. Every time the linear motor impact on the surface of PVDF-TrFE devices, it may generate a piezoelectric response evidenced by the sharp voltage and current pulse peak. For the open-circuit voltage, the voltage pulse peak contains a positive peak and a larger negative peak. Since the PVDF-TrFE films were polarized in one direction, the negative peak is always lager than the positive peak. Besides, with the accelerated speed increasing from 0.1 to $5 \mathrm{~m} / \mathrm{s}^{2}$, pressure increased from 0.1 to $5 \mathrm{~N}$, and the output voltage increased from 2.0 to $8.7 \mathrm{~V}$ because the higher accelerated speed impact may lead to larger deformation of the $\beta$-phase crystal.

As for the current, positive and negative pulses are generated by the direct impact and relaxed stress from the damping effect, respectively. In Figure $\mathbf{8 B}$, the PVDF-TrFE devices generate a positive current between 40 and $656 \mathrm{nA}$ and a negative current ranging from 58 to $480 \mathrm{nA}$, with the accelerated speed increasing from 0.1 to $5 \mathrm{~m} / \mathrm{s}^{2}$. From the voltage and the current output 
curves, it was found that the difference between positive and negative voltage is greater than the difference between the current peaks. Since the PVDF-TrFE device equals to a capacitor, the induced charges will be stored on the surface of the electrodes when they are generated. These induced charges will counteract as the opposite voltage is applied. Therefore, the negative voltage peaks are larger than the positive ones. As for the short-circuit current, it only depends on the impact of accelerated speed. The output current generated by direct impact and the relaxed stress has no significant difference. Therefore, the positive and negative current peaks almost have no differences. At the same time, we also tested the current density and power density of the PVDFTrFE piezoelectric device under different forces and loads, as shown in Figure 8C and Figure 8D. The current density increases from $2 \mathrm{nA} / \mathrm{cm}^{2}$ to $32.8 \mathrm{nA} / \mathrm{cm}^{2}$ as the force increases, because the greater the force, the greater the deformation of the crystal. For the power density, the maximum power density is $9.78 \mathrm{~mW} / \mathrm{cm}^{3}$ when the load is $80 \mathrm{M} \Omega$.

\section{CONCLUSION}

In this study, PVDF-TrFE films with a large area of $200 \mathrm{~mm}^{2} \times$ $200 \mathrm{~mm}^{2}$, high $\mathrm{d}_{33}$ coefficient, and uniform distribution of $25 \pm$ $2 \mathrm{pC} / \mathrm{N}$ were prepared by in situ polarization. The in situ polarization was performed with low poling voltage, and short

\section{REFERENCES}

AlamusiXue, J., Wu, L., Hu, N., Qiu, J., Chang, C., et al. (2012). Evaluation of Piezoelectric Property of Reduced Graphene Oxide (rGO)-Poly(vinylidene Fluoride) Nanocomposites. Nanoscale 4, 7250-7255. doi:10.1039/c2nr32185h

Bae, J.-H., and Chang, S.-H. (2015). Characterization of an Electroactive Polymer (PVDF-TrFE) Film-type Sensor for Health Monitoring of Composite Structures. Compos. Structures 131, 1090-1098. doi:10.1016/ j.compstruct.2015.06.075

Banik, N. C., Boyle, F. P., Sluckin, T. J., Taylor, P. L., Tripathy, S. K., and Hopfinger, A. J. (1980). Theory of Structural Phase Transitions in Crystalline Poly(vinylidene Fluoride). J. Chem. Phys. 72, 3191-3196. doi:10.1063/1.439552

Bhavanasi, V., Kumar, V., Parida, K., Wang, J., and Lee, P. S. (2015). Enhanced Piezoelectric Energy Harvesting Performance of Flexible PVDF-TrFE Bilayer Films with Graphene Oxide. ACS Appl. Mater. Inter. 8, 521-529. doi:10.1021/ acsami.5b09502

Bune, A. V., Fridkin, V. M., Ducharme, S., Blinov, L. M., Palto, S. P., Sorokin, A. V., et al. (1998). Two-dimensional Ferroelectric Films. Nature 391, 874-877. doi: $10.1038 / 36069$

Chan, H., Chan, W., and Choy, C. (2003). Piezoelectric and Pyroelectric Properties of PT/P(VDF-TrFE) 0-3 Composites. Ferroelectrics Lett. 2 (3), 123-127. doi:10.1080/07315178408200576

Chen, Y.-C., Cheng, C.-K., and Shen, S.-C. (2019). Design and Fabrication of a Displacement Sensor Using Screen Printing Technology and Piezoelectric Nanofibers in D33 Mode. Sens. Mater. 31, 233. doi:10.18494/SAM.2019.2083

Cheng-Lu, L., Zhong-Hai, M., Qi, X., Bao, R. Y., Yang, W., Xie, B. H., et al. (2014). Induced Formation of Dominating Polar Phases of Poly(vinylidene Fluoride): Positive Ion-CF2 Dipole or Negative Ion-CH2 Dipole Interaction. J. Phys. Chem. B 118 (30), 9104-9111. doi:10.1021/jp504938f

Dai, X., Niu, J., Ren, Z., Sun, X., and Yan, S. (2016). Effects of Nanoporous Anodic Alumina Oxide on the Crystallization and Melting Behavior of Poly(vinylidene Fluoride). J. Phys. Chem. B 120, 843-850. doi:10.1021/acs.jpcb.5b11178 poling time compared with traditional poling methods, which enable the quantity production of PVDF-TrFE films in industrial and commercial applications. Besides, the XRD and DSC results proved the in situ polarization can promote the transition from the $\alpha$-phase crystal to the $\beta$-phase crystal and the formation of the $\beta$-phase crystal in the PVDF-TrFE film, which will further improve its piezoelectricity. Thus, the high efficiency, easy processing, and the excellent and uniform $\mathrm{d}_{33}$ value of PVDFTrFE films make the in situ polarization a great potential in piezoelectric material processing methods.

\section{DATA AVAILABILITY STATEMENT}

The original contributions presented in the study are included in the article/Supplementary Material; further inquiries can be directed to the corresponding author.

\section{AUTHOR CONTRIBUTIONS}

$\mathrm{XH}$ : conceptualization and writing original draft. MY: methodology and investigation. NY: methodology and data curation. XZ: conceptualization and supervision. YX: funding acquisition. All authors contributed to review and editing of the manuscript.

Davis, G. T., Mckinney, J. E., Broadhurst, M. G., and Roth, S. C. (1978). Electricfield-induced Phase Changes in Poly(vinylidene Fluoride). J. Appl. Phys. 49, 4998-5002. doi:10.1063/1.324446

Dietze, M., and Es-Souni, M. (2008). Structural and Functional Properties of Screen-Printed PZT-PVDF-TrFE Composites. Sensors Actuators A: Phys. 143, 329-334. doi:10.1016/j.sna.2007.11.016

Fadzallah, I. A., Sabran, N. S., Van Toan, N., Ono, T., Mohd Said, S., and Mohd Sabri, M. F. (2020). Micropatterning and Integration of Electrospun Pvdf Membrane into Microdevice. J. Microelectromech. Syst. 29 (99), 438-445. doi:10.1109/JMEMS.2020.2983717

Fukada, E., and Sakurai, T. (1971). Piezoelectricity in Polarized Poly(vinylidene Fluoride) Films. Polym. J. 2, 656-662. doi:10.1295/polymj.2.656

Furukawa, T. (1989). Ferroelectric Properties of Vinylidene Fluoride Copolymers. Phase Transitions 18 (3-4), 143-211. doi:10.1080/01411598908206863

Hasegawa, R., Takahashi, Y., Chatani, Y., and Tadokoro, H. (1972). Crystal Structures of Three Crystalline Forms of Poly(vinylidene Fluoride). Polym. J. 3, 600-610. doi:10.1295/polymj.3.600

Hu, Y., Xu, P., Gui, H., Yang, S., and Ding, Y. (2015). Effect of Graphene Modified by a Long Alkyl Chain Ionic Liquid on Crystallization Kinetics Behavior of Poly(vinylidene Fluoride). RSC Adv. 5, 92418-92427. doi:10.1039/ C5RA17169E

Karan, S. K., Bera, R., Paria, S., Das, A. K., Maiti, S., Maitra, A., et al. (2016). An Approach to Design Highly Durable Piezoelectric Nanogenerator Based on Self-Poled PVDF/AlO-rGO Flexible Nanocomposite with High Power Density and Energy Conversion Efficiency. Adv. Energ. Mater. 6, 1601016. doi:10.1002/ aenm.201601016

Koten, V. G., Polm, L. H., Elsevier, C. J., Ernsting, J. M., Stufkens, D. J., Vrieze, K., et al. (1987). Synthesis of [Ru2(CO)5\{1,2-bis(m-alkylamido)-1,2-bis(2-pyridyl) ethane\}] and Ru2. Organometallics 6, 1096-1104. doi:10.1021/om00148a031

Li, C., Wu, P. M., Lee, S., Gorton, A., Schulz, M. J., and Ahn, C. H. (2008). Flexible Dome and Bump Shape Piezoelectric Tactile Sensors Using PVDF-TrFE Copolymer. J. Microelectromech. S 17, 334-341. doi:10.1109/ JMEMS.2007.911375 
Liu, X., Deng, M., and Wang, X. (2017). Nanoscale Domain Imaging and Local Piezoelectric Coefficient D33 Studies of Single Piezoelectric Polymeric Nanofibers. Mater. Lett. 189, 66-69. doi:10.1016/j.matlet.2016.11.044

Lovinger, A. J. (1983). Ferroelectric Polymers. Science 220, 1115-1121. doi:10.1126/ science.220.4602.1115

Lovinger, A. J., Furukawa, T., Davis, G. T., and Broadhurst, M. G. (1983). Crystallographic Changes Characterizing the Curie Transition in Three Ferroelectric Copolymers of Vinylidene Fluoride and Trifluoroethylene: 2. Oriented or Poled Samples. Polymer 24, 1233-1239. doi:10.1016/00323861(83)90051-4

Lutkenhaus, J. L., Mcennis, K., Serghei, A., and Russell, T. P. (2010). Confinement Effects on Crystallization and Curie Transitions of Poly(vinylidene Fluoride-CoTrifluoroethylene). Macromolecules 43, 3844-3850. doi:10.1021/ma100166a

Masahiko, T., Sumio, H., Susumu, M., and Nobuyuki, O. (1977). Some Aspects of Piezoelectricity and Pyroelectricity in Uniaxially Stretched Poly(vinylidene Fluoride). J. Appl. Phys. 48, 513. doi:10.1063/1.323695

Mcginn, C. K., Kam, K. A., Laurila, M.-M., Lozano Montero, K., Mäntysalo, M., Lupo, D., et al. (2020). Formulation, Printing, and Poling Method for Piezoelectric Films Based on PVDF-TrFE. J. Appl. Phys. 128 (22), 225304. doi:10.1063/5.0027855

Moyle, B. D. (1969). Piezoelectricity in Poly (Vinylidene Fluoride). Jpn. J. Appl. Phys. 8, 975. doi:10.1143/JJAP.8.975

Nan, J., Qian, H., Jing, S., Guangmei, X., and Rui, S. (2017). Crystallization Behavior and Electroactive Properties of PVDF, P(VDF-TrFE) and Their Blend Films. Polym. Test. 57, 302-306. doi:10.1016/j.polymertesting.2016.12.003

Ohigashi, H., Omote, K., and Gomyo, T. (1995). Formation of "single Crystalline Films" of Ferroelectric Copolymers of Vinylidene Fluoride and Trifluoroethylene. Appl. Phys. Lett. 66, 3281-3283. doi:10.1063/1.113730

Pi, Z., Zhang, J., Wen, C., Zhang, Z.-b., and Wu, D. (2014). Flexible Piezoelectric Nanogenerator Made of Poly(vinylidenefluoride-Co-Trifluoroethylene) (PVDFTrFE) Thin Film. Nano Energy 7, 33-41. doi:10.1016/j.nanoen.2014.04.016

Prest, W. M., and Luca, D. J. (1978). The Formation of the $\gamma$ Phase from the $\alpha$ and $\beta$ Polymorphs of Polyvinylidene Fluoride. J. Appl. Phys. 49, 5042-5047. doi:10.1063/1.324439

Sajkiewicz, P., Wasiak, A., and Gocłowski, Z. (1999). Phase Transitions during Stretching of Poly(vinylidene Fluoride). Eur. Polym. J. 35, 423-429. doi:10.1016/S0014-3057(98)00136-0

Sencadas, V., Gregorio, R., and Lanceros-Méndez, S. (2009). a to $\beta$ Phase Transformation and Microestructural Changes of PVDF Films Induced by Uniaxial Stretch. J. Macromolecular Sci. B 48, 514-525. doi:10.1080/ 00222340902837527
Sharma, M., Sharma, K., and Bose, S. (2013). Segmental Relaxations and Crystallization-Induced Phase Separation in PVDF/PMMA Blends in the Presence of Surface-Functionalized Multiwall Carbon Nanotubes. J. Phys. Chem. B 117, 8589-8602. doi:10.1021/jp4033723

Wang, Z.-Y., Fan, H.-Q., Su, K.-H., Wang, X., and Wen, Z.-Y. (2007). Structure, Phase Transition and Electric Properties of Poly(vinylidene FluorideTrifluoroethylene) Copolymer Studied with Density Functional Theory. Polymer 48, 3226-3236. doi:10.1016/j.polymer.2007.04.015

Won, K., Han, M. H., Shin, Y. H., Kim, H., and Lee, E. K. (2016). First-Principles Study of the $\alpha-\beta$ Phase Transition of Ferroelectric Poly(vinylidene Difluoride): Observation of Multiple Transition Pathways. J. Phys. Chem. B 120, 3240-3249. doi:10.1021/acs.jpcb.6b00881

Xue, L., Lim, Y. F., Yao, K., Tay, F., and Seah, K. H. (2013). Ferroelectric Poly(vinylidene Fluoride) Homopolymer Nanotubes Derived from Solution in Anodic Alumina Membrane Template. Chem. Mater. 25, 524-529. doi:10.1021/cm3028466

Yaqoob, U., and Chung, G.-S. (2017). Effect of Reduced Graphene Oxide on the Energy Harvesting Performance of P(VDF-TrFE)-BaTiO3 Nanocomposite Devices. Smart Mater. Struct. 26, 095060. doi:10.1088/1361-665X/aa81a0

Ying, W., Zhenyu, L., Xiuyi, K., Xinying, S., Xu, L., Zhenyu, W., et al. (2015). Exceptional Dielectric Properties of Chlorine-Doped Graphene Oxide/poly (Vinylidene Fluoride) Nanocomposites. Carbon: Int. J. Sponsored by Am. Carbon Soc. 89, 102-112. doi:10.1016/j.carbon.2015.02.074

Conflict of Interest: The authors declare that the research was conducted in the absence of any commercial or financial relationships that could be construed as a potential conflict of interest.

Publisher's Note: All claims expressed in this article are solely those of the authors and do not necessarily represent those of their affiliated organizations, or those of the publisher, the editors and the reviewers. Any product that may be evaluated in this article, or claim that may be made by its manufacturer, is not guaranteed or endorsed by the publisher.

Copyright (c) $2021 \mathrm{Hu}$, You, Yi, Zhang and Xiang. This is an open-access article distributed under the terms of the Creative Commons Attribution License (CC BY). The use, distribution or reproduction in other forums is permitted, provided the original author(s) and the copyright owner(s) are credited and that the original publication in this journal is cited, in accordance with accepted academic practice. No use, distribution or reproduction is permitted which does not comply with these terms. 